\title{
Contamination of used toothbrushes and their decontamination with disinfecting agents
}

SADJ October 2020, Vol. 75 No. 9 p478 - p484

TRMD Ralephenya ${ }^{1}, \mathrm{~J} \mathrm{Molepo}^{2}$, M Molaudzi $^{3}$, A Volchansky $^{4}$, SL Shangase ${ }^{5}$

\section{ABSTRACT}

Aim

To evaluate microbial contamination of toothbrushes and the efficacy of different oral disinfectant agents in their decontamination.

\section{Methods}

The heads of 98 used toothbrushes derived from patients who needed oral hygiene treatment were included in the study. In the laboratory, microorganisms on toothbrush heads were identified using standard microbiological methods. Toothbrush heads with microorganisms were then randomly divided into four groups of 19 and disinfected with Brushtox, Andolex $\mathrm{C}$, Listerine ${ }^{\circledR}$ and water (control), followed by microbiological analysis.

\section{Results}

Seventy eight percent of toothbrushes were contaminated with different microorganisms. Coagulase-negative staphylococci (CoNS) were found in a high number of toothbrushes (49\%), followed by Pseudomonas spp (37\%), Staphylococcus aureus (32\%), Streptococcus

Author affiliations:

1. Tshakane RMD Ralephenya: Diploma in Oral Hygiene, MA (Development Studies), Lecturer, Division of Oral Hygiene and Dental Therapy, School of Oral Health Sciences, Faculty of Health Sciences, University of the Witwatersrand, Johannesburg, South Africa.

ORCID Number: 0000-0002-0117-2942

2. Julitha Molepo: $P h D$ (Medical Microbiology), Head, Department of Oral Biological Sciences, School of Oral Health Sciences, Faculty of Health Sciences, University of the Witwatersrand, Johannesburg, South Africa.

3. Mulalo Molaudzi: MSc (Medical Microbiology), Lecturer, Department of Oral Biological Sciences, School of Oral Health Sciences, Faculty of Health Sciences, University of the Witwatersrand, Johannesburg, South Africa.

4. Alfred Volchansky: $P h D$, Consultant, Department of Oral Medicine and Periodontology, School of Oral Health Sciences, Faculty of Health Sciences, University of the Witwatersrand, Johannesburg, South Africa.

5. Sindisiwe L Shangase: M Dent, Head, Department of Oral Medicine and Periodontology, School of Oral Health Sciences, Faculty of Health Sciences, University of the Witwatersrand, Johannesburg, South Africa.

Corresponding author: Tshakane RMD Ralephenya

University of the Witwatersrand, Johannesburg, Faculty of Health

Sciences, School of Oral Health Sciences, 7 York Road, Parktown.

Email: tshakane.ralephenya@wits.ac.za

Author contributions:

1. Tshakane RMD Ralephenya: Principal author - $30 \%$

Julitha Molepo: Co-author - $25 \%$

Mulalo Molaudzi: Co-author - $25 \%$

Alfred Volchansky: Co-author - 10\%

Sindisiwe L Shangase: Co-author - 10\% mutans (14\%), coliforms (9\%) and Candida albicans (3\%). Decontamination of toothbrushes with Andolex $\mathrm{C}$ and Listerine ${ }^{\circledR}$ reduced the number of contaminated toothbrushes by $74 \%$ each, Brushtox by $90 \%$ and water by $0.0 \%$.

\section{Conclusion}

Toothbrushes were contaminated with various types of microorganisms, but predominantly with CoNS. Although Brushtox is a toothbrush spray, soaking toothbrushes in this solution was the most effective method, reducing the number of disinfected toothbrushes by $90 \%$. Oral health practitioners should raise awareness to their patients regarding the need to disinfect toothbrushes.

\section{Keywords}

Toothbrush, microorganisms, contamination, oral disinfectant agents.

\section{INTRODUCTION}

Toothbrushes are the most commonly used oral hygiene tools to remove plaque and maintain good oral health., ${ }^{1,2}$ Oral disinfectant agents could be utilized to supplement or even supplant patient-dependent mechanical plaque control and thus reduce or prevent oral disease. ${ }^{3}$ Microorganisms from the oral cavity such as Streptococcus, Staphylococcus, and Lactobacilli could contaminate them during brushing. ${ }^{4}$

These micro-organisms may cause oral, respiratory, gastrointestinal, cardiovascular and renal diseases. . $^{5,6,7}$ Organisms associated with periodontal diseases ${ }^{8,9}$ and opportunistic pathogens such as Candida albicans ${ }^{10}$ have also been implicated in toothbrush contamination. The possibility of infection/re-infection with resultant disease manifestation, because of the contamination of toothbrushes by microorganisms is a cause for concern.

Storage of toothbrushes in bathrooms is a common practice worldwide including South Africa, and plays a role in their contamination. Bathrooms often have attached toilets, thus increasing the chances of toothbrush contamination by enteric bacteria such as Escherichia coli. ${ }^{4}$ In an attempt to limit the contamination, toothbrushes in some households are stored in covers. However, such practice facilitates retention of moisture 
thus prolonging drying time, which may lead to proliferation of opportunistic microorganisms such as Pseudomonas aeruginosa. ${ }^{11}$

The American Dental Association (ADA) recommends replacement of toothbrushes every three to four months of use to maintain the effectiveness of the brushes in removing dental plaque biofilm. ${ }^{12}$ Most oral health practitioners have adopted this and recommend it to their patients. However, in developing countries where resources are limited, the majority of people may find it costly, and therefore impractical to replace toothbrushes every three to four months.

Whilst regular toothbrush change may enhance removal of dental plaque biofilm, it does not completely address the problem of contamination, as new toothbrushes were found to harbor significantly more Streptococcus mutans than worn toothbrushes. ${ }^{13}$ It is of even greater concern that new unused toothbrushes from different manufacturers may present with bacterial contamination before oral contact. ${ }^{5,14}$

Previous studies have investigated and reported microbial contamination of toothbrushes after use. ${ }^{15,16,17,18,19,20}$ Contaminated toothbrushes may lead to oral and systemic diseases, such as gastrointestinal, cardiovascular, respiratory, renal problems and septicaemia. ${ }^{21}$ There is an important need to disinfect toothbrushes to prevent these diseases, especially in children, the elderly and immunosuppressed individuals including those undergoing organ transplants or chemotherapy. ${ }^{19}$

Various methods have been evaluated for the disinfection of toothbrushes including antiseptic rinses or sprays, coating toothbrush bristles with toothpaste or gel with antimicrobial effect. ${ }^{9,22,23,24,15}$ There are only a few studies on toothbrush disinfection using Brushtox and Listerine ${ }^{\circledR}$ mouth rinse and no documented studies on the use of Andolex mouth rinse as a toothbrush disinfectant.

Hence, the aim of this study was to evaluate the microbial contamination of toothbrushes and the efficacy of Andolex $\mathrm{C}$, Listerine ${ }^{\circledR}$ and Brushtox in disinfecting the contaminated toothbrushes as a strategy to prevent oral diseases in a South African population.

\section{MATERIALS AND METHODS}

\section{Study population}

Patients of both genders attending the Wits Oral Health Centre for oral hygiene treatment were recruited to participate in the study. Patients were informed about the study at a screening appointment. At this visit, they were given both a written and an oral explanation of the background of the study, its objectives, and their involvement.

Signed consent forms were obtained from all patients who agreed to participate in the study and they were asked to bring the toothbrush that they have been using in the past three months. Patients that were included had to be a minimum of 18 years of age, not having systemic diseases such as diabetes mellitus and hypertension which was considered as healthy, not having used antibiotics, antifungal agents and mouth rinses within the 30-day period prior to data and toothbrush collection and not having had any form of periodontal therapy in the past 3 months. Medical history of the participants was obtained from the participants themselves to rule out systemic diseases. The study was approved by the Human Research Ethics Committee (Medical) of the University of the Witwatersrand, Johannesburg (M120290).

\section{Methodology}

A total of 98 used manual toothbrush heads were included in this study. Used toothbrushes of different designs were collected from patients and immediately placed into sterile pouches and labelled. Convenience sampling method was used to collect toothbrushes. The toothbrushes were not exposed to each other during the time of collection. All toothbrushes were processed within 24 hours of collection. The handle of the used toothbrush was aseptically cut off using a rotary saw and retained in the bag to avoid contamination. ${ }^{25}$

Each toothbrush head was soaked for 5 hours in a separate tube containing $15 \mathrm{ml}$ of sterile tryptone soya broth (TSB) medium. Following soaking, the contents were vortexed for 60 seconds and hundred microlitres of the resulting suspension was plated onto MacConkey agar, Mitis salivarius Bacitracin (MSB) agar and Sabouraud's Dextrose agar (SDA) to select for enterobacteria, mutans streptococci, and yeasts, respectively. All plates were incubated aerobically at $370 \mathrm{C}$ for 48 hours except for MSB agar, which was incubated for 48 hours under $\mathrm{CO}_{2}$ conditions in a candle jar.

After incubation, the colour and morphology of colonies were recorded, and Gram's staining was performed on a representative of each colony morphotype. Coagulase and catalase tests were performed to confirm and differentiate Staphylococci. Colonies from SDA were identified by wet mount and ID32C test following the manufacturer's instructions (bioMérieux, Marcy-L'Etoile, France).

Streptococcus mutans was identified morphologically from MSB agar as convex, pale blue colonies with granular frosted glass appearance. Gram-negative bacilli were subcultured on MacConkey agar and tested for oxidase (Pro-Lab Diagnostics, Basingstoke, UK).

Gram-negative, non-lactose-fermenting, oxidase-positive colonies isolated on MacConkey agar were considered to be Pseudomonas spp, while Gram-negative, lactosefermenting, oxidase negative colonies isolated on MacConkey agar were considered to be coliforms. For every test conducted, positive and negative controls were included to ensure accuracy and validity of results.

In the decontamination procedure, the toothbrush heads that showed growth were randomly divided into four groups of 19 and immersed overnight in $15 \mathrm{ml}$ each of the following oral disinfectant agents: Andolex $\mathrm{C}$ (Chlorhexidine gluconate 0.12\%; iNova Pharmaceuticals (Pty) Ltd, Bedfordview, South Africa), Brushtox (Chlorhexidine gluconate 0.2\%; Dentox Ltd., Warwick, England), Listerine $\AA$ (with 26.98\% alcohol; Johnson \& Johnson, New Jersey, USA), and sterile distilled water (control). 
Toothbrush heads were then rinsed with sterile distilled water to wash off excess oral disinfectant agents, placed in $15 \mathrm{ml}$ of sterile TSB medium for 5 hours and the resulting suspension cultured on the same media as above. Standard microbiological methods were repeated as above to identify the different organisms.

\section{Statistical analysis}

Disinfection data was analysed using the Kruskal-Wallis ANOVA test. All statistical significance was calculated at the $5 \%$ significance level.

\section{RESULTS}

Our data showed that microorganisms were present on $76(78 \%)$ of the 98 tested toothbrushes. No growth was observed in the remaining 22 toothbrushes. Coagulasenegative staphylococci (CoNS) were found in a high number of toothbrushes ( $n=37 ; 49 \%)$, followed by Pseudomonas spp $(n=28 ; 37 \%)$, Staphylococcus aureus $(n=24 ; 32 \%)$, Streptococcus mutans $(n=11 ; 14 \%)$, Coliforms ( $n=7 ; 9 \%)$ and Candida albicans $(n=2 ; 3 \%)$ (Figure 1).

After using the various oral disinfectant agents, Andolex $C$ and Listerine ${ }^{\circledR}$ reduced the number of contaminated toothbrushes by $74 \%(n=14)$ and Brushtox by $90 \%$ $(n=17)$. Water could not disinfect any of the contaminated toothbrushes (Figure 2).

\section{Statistical analysis}

There was no statistically significant difference when Brushtox was compared with Listerine $\AA$ and Andolex $C$ in the disinfection of toothbrushes $(p=0.4048)$. Statistically significant differences were observed in disinfection efficacy between Brushtox, Listerine $\AA$ and Andolex $\mathrm{C}$ when compared to sterile distilled water $(p<0.00001)$.

\section{DISCUSSION}

Toothbrushes are the most common tools used to improve the oral health of an individual. The ADA recom mends that toothbrushes be replaced every three to four months of use to maintain the effectiveness of the brushes in removing dental plaque biofilm. ${ }^{12}$ Evidence that used toothbrushes retain oral microorganisms has been previously demonstrated from countries other than South Africa.

Toothbrushes are the most common tools used to improve the oral health of an individual. The ADA recommends that toothbrushes be replaced every three to four months of use to maintain the effectiveness of the brushes in removing dental plaque biofilm. ${ }^{12}$

Evidence that used toothbrushes retain oral microorganisms has been previously demonstrated from countries other than South Africa ${ }^{3,18,25}$, however, there is very little public awareness that toothbrushes can be contaminated with use.

Although various methods have been evaluated for the disinfection of toothbrushes, $, 915,22,23,24$ the use of Andolex

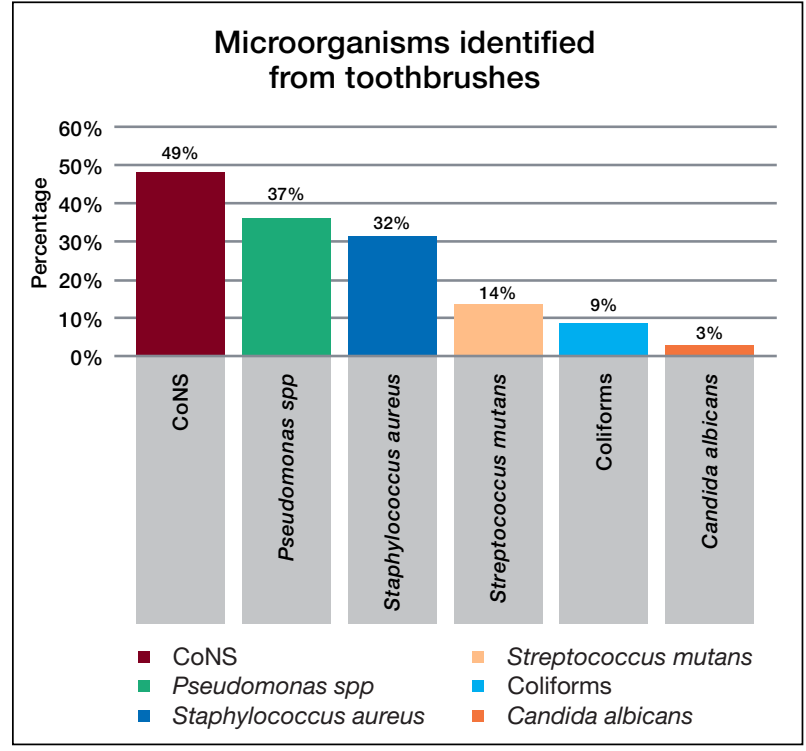

Figure 1. Microorganisms identified from toothbrushes $(N=76)$.

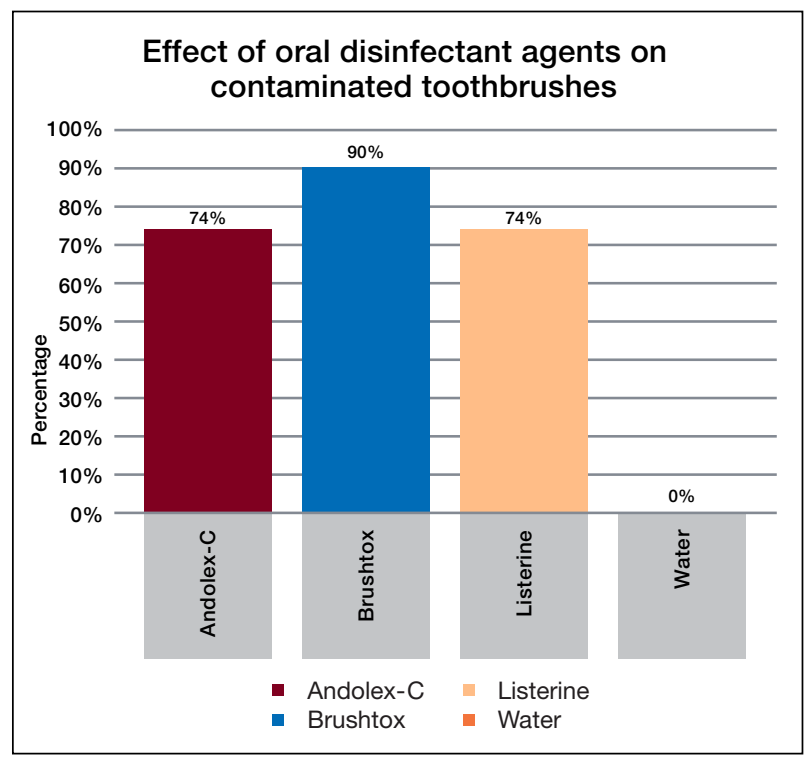

Figure 2. Effect of oral disinfectant agents on contaminated toothbrushes.

C, Brushtox and Listerine ${ }^{\circledR}$ as oral disinfectant agents has not been widely studied.

Hence, the aim of this study was to evaluate the microbial contamination of toothbrushes and the efficacy of Andolex C, Brushtox and Listerine ${ }^{\circledR}$ in disinfecting the contaminated toothbrushes as a strategy to prevent oral diseases in a South African population.

In the current study, 76 (79\%) of tested toothbrushes showed microbial contamination. The microorganisms isolated were coagulase-negative staphylococci (CoNS), Pseudomonas spp, Staphylococcus aureus, Streptococcus mutans, coliforms and Candida albicans. These results confirm that toothbrushes may play a major role in the retention of organisms which may pose a health risk in susceptible individuals.

These toothbrushes were collected from patients seeking oral treatment from infections which could have been caused by these contaminated toothbrushes. 
The absence of growth in 22 toothbrushes from this study could have been due to participants submitting new, purchased toothbrushes instead of the ones they used at home or they could have disinfected the toothbrushes before submission. In addition, the media used for culture were specific for growth of enterobacteria, mutans streptococci, and yeasts, therefore these 22 toothbrushes could be having other pathogens not tested in this study.

The results of our study are in concordance with those of previous studies. Mehta et al. ${ }^{11}$ and Ferreira et $\mathrm{al}^{26}$ reported that $70 \%$ of the toothbrushes were heavily contaminated with different pathogenic microorganisms after use. Pesevska et al. ${ }^{17}$ and Rodrigues et al. ${ }^{19}$ evaluated microbial contamination of toothbrushes used by Dentistry students and microbial growth was observed in $100 \%$ and $91 \%$ of toothbrushes respectively, thus increasing the risk of oral diseases in this healthy population. These investigators reported that contamination of toothbrushes occurs often, even in individuals who should know the ideal conditions for the storage of toothbrushes.

In this study, coagulase-negative staphylococci (CoNS) were the most predominant organisms found in the highest number of toothbrushes (49\%). In contrast, Ferreira et al. ${ }^{26}$ found CoNS to be the least isolated organisms (10\%) in toothbrushes of healthy individuals whilst Saini and Kulkarni ${ }^{27}$ isolated CoNS from $36 \%$ of toothbrushes obtained from a similar population.

CoNS are normal inhabitants of human skin and mucous membranes ${ }^{27}$ and their predominant occurrence in toothbrushes in the current study may be due to handling and rinsing of the toothbrushes after use ${ }^{27}$. However, the pathogenic potential of the predominant CoNS cannot be ignored as these microorganisms have been implicated in various infections including endocarditis, bacteraemia, surgical site and urinary tract infections ${ }^{28}$. In addition, CoNS have been reported to be multidrug resistant, which could lead to difficulty in treating infections caused by these organisms ${ }^{29}$.

Pseudomonas spp. was found in $37 \%$ of toothbrushes and was the second most isolated organism. Osho et al. ${ }^{30}$ found Pseudomonas in $40 \%$ of toothbrushes collected from healthy patients and concluded that toothbrushes can serve as fomites in various homes especially if they are not properly used and taken care of. On the contrary, Pesevska et al. ${ }^{17}$ reported Pseudomonas in $15 \%$ of toothbrushes from healthy students and indicated that toothbrushes can be carriers of microorganisms, increasing the risk of diseases in healthy people.

Another study found Pseudomonas in $83 \%$ of toothbrushes from healthy individuals and those with oral infections. ${ }^{31}$ Pseudomonas spp causes various infections including respiratory system infections, soft tissue infections, bacteraemia, and a variety of systemic infections, particularly in immunocompromised patients. These micro-organisms can survive in water therefore their presence in toothbrushes may be due to incorrect storing of the toothbrushes, where the aerosols from the toilet could easily reach them. ${ }^{32}$
In this study, Staphylococcus aureus (S. aureus) was isolated in $32 \%$ of toothbrushes. Contrary to this finding, Abd-ulnabi ${ }^{31}$ reported S. aureus in $21 \%$ of toothbrushes from individuals with oral infections but not from healthy individuals and concluded that toothbrushes may be a source of opportunistic pathogens such as $S$. aureus due to their incorrect storage. Similarly Osho et al. ${ }^{30}$ found S. aureus in $20 \%$ of toothbrushes of the same brand and type used by different individuals.

S. aureus was the most predominant isolated organism in $60 \%$ of toothbrushes used twice per day by students. ${ }^{34} \mathrm{~S}$. aureus was found in the lowest number (10\%) of toothbrushes collected from students in a study by Osungunna and Oyajoju. ${ }^{34}$ This microorganism has been implicated in life threatening diseases including infective endocarditis and bacteraemia and can influence the occurrence of oral diseases such as dental caries and gingivitis. ${ }^{10,15}$ In addition, S. aureus has been reported to develop resistance to several antibiotics including methicillin (MRSA), penicillin and cephalosporins. ${ }^{36}$ In cases where toothbrushes are shared, MRSA can be transmitted from the infected individuals to the uninfected, thus requiring that the toothbrushes be disinfected regularly.

Streptococcus mutans (S. mutans) was isolated in $14 \%$ of tested toothbrushes. These results differ from those of a study by Rodrigues et al..$^{19}$ where S. mutans was detected in $47 \%$ of toothbrushes, highlighting the ability of this organism to form biofilms and binding to the material of the toothbrushes. Saini and Kulkarni ${ }^{27}$ reported S. mutans in 36\% of toothbrushes from healthy individuals and concluded that toothbrushes are considered a potential biohazard for reintroduction of microorganisms in the oral cavity. Efstratiou et al. ${ }^{36}$ found that immediately after brushing, 30\% of toothbrushes were contaminated with S. mutans.

The presence of $S$. mutans in toothbrushes in the current study is not surprising as they are part of the oral normal flora. The growth of these bacteria on toothbrushes may be due to improper rinsing of toothbrushes after use. S. mutans is currently considered to be the major cause of dental caries ${ }^{37}$ due to its exceptional aciduric and acidogenic properties and the use of a contaminated toothbrush may lead to reinfection with this organism, escalating dental caries problem.

Coliforms, which are organisms not normally associated with oral flora were isolated in $9 \%$ of tested toothbrushes. Similarly, Osho et al. ${ }^{30}$ reported Escherichia coli (E. coli) in $10 \%$ of tested toothbrushes from different individuals. In contrast, Samuel et al. ${ }^{33}$ reported E. coli in $20 \%$ of toothbrushes from students whilst Sammons et $\mathrm{al}^{24}$ showed that $28 \%$ of toothbrushes from healthy volunteers were contaminated with E. coli.

A study by Ferreira et al. ${ }^{26}$ found that $80 \%$ of toothbrushes from healthy individuals were contaminated with E. coli. These investigators found that $90 \%$ of the toothbrushes were stored in bathrooms which could have accounted for the high contamination with these faecal bacteria. 
Coliforms are normally found in the gastrointestinal tract and therefore in faecal matter. Aerosols released after flushing the toilet can deliver faecal matter up to 20 feet in the air, reaching toothbrushes that have been stored on bathroom counters. ${ }^{38}$ The presence of coliforms in toothbrushes in the current study shows faecal contamination. In addition, these bacteria may have entered the toothbrushes through the rinsing water as coliforms can be found in water. ${ }^{39}$ In addition to gastrointestinal tract infections, coliforms have been shown to cause respiratory tract infections. ${ }^{38,40}$

Candida albicans (C. albicans) was isolated in a low number of toothbrushes (3\%) in agreement with a study by Pesevska et al. ${ }^{17}$ where C. albicans was reported in $5 \%$ of toothbrushes. In contrast, in an in vitro study, Nascimento et al. ${ }^{19}$ reported C. albicans in $37.3 \%$ of toothbrushes after treatment with water, showing that rinsing toothbrushes with water does not disinfect the toothbrushes. Wetzel et al. ${ }^{10}$ reported that toothbrushes made with different anchoring techniques could retain C. albicans. Another study reported that contamination of toothbrushes with Candida was significantly higher in the samples of diseased individuals compared to healthy individuals. ${ }^{41} \mathrm{C}$. albicans may colonize the mucosal surfaces including periodontal tissues, especially in immunocompromised individuals and patients with extended antibiotic treatment. ${ }^{42}$ The source of the C. albicans in the current study could have most likely been the oral cavity where it is a normal flora.

Although C. albicans was isolated in low numbers in this study, its presence is significant as infection with C. albicans has been shown to be an important cause of mortality and morbidity in patients with AIDS, bone marrow transplantation and aggressive anti-neoplastic therapy. ${ }^{43}$

The presence of the microorganisms in the toothbrushes in this study highlights the need to control the microbial contamination of toothbrushes in order to prevent reinfection of the mouth and dissemination of microorganism to other parts of the body.

In our study, overnight immersion of contaminated toothbrushes in Brushtox (CHX gluconate 0.2\%) was found to be highly effective in disinfecting $90 \%$ of the toothbrushes. These results are in agreement with those of an in vitro study by Neal et al. ${ }^{44}$ where Brushtox showed $100 \%$ efficacy against bacteria and fungi on toothbrushes. Macari et al. ${ }^{45}$ corroborated these findings, with Brushtox reported as the best disinfectant for prevention of cariogenic biofilm formation on $84.4 \%$ of children's toothbrushes. These results are in contrast with those of a previous clinical study where Brushtox was less effective than $0.12 \%$ chlorhexidine in removing S. mutans biofilms from toothbrush bristles. ${ }^{46}$

Brushtox Antiseptic Toothbrush Cleanser (Dentox Limited, Warwick, England) is a chemical solution that was developed for toothbrush disinfection. Brushtox consists of activated ethanol solution (35-40\%), 4.5\% biocides and a mixture of para-hydroxybenzoate, and is highly effective against a wide range of bacteria, fungi and viruses. ${ }^{47}$ The results of our study agree with this as
Brushtox decontaminated $90 \%$ of the toothbrushes contaminated with bacteria and fungi.

Andolex mouth rinse $(0.12 \%$ chlorhexidine) was found to be less effective than Brushtox in the current study. When reviewing the literature, no documented studies on the use of Andolex mouth rinse as a toothbrush disinfectant could be found. However, a study by Rodrigues et al. ${ }^{19}$ reported a significant decrease (91\%) in toothbrushes contaminated with Streptococci, Streptococcus mutans, Staphylococci, Enterobacteria and yeast, when they were sprayed with $0.12 \%$ chlorhexidine solution three times a day after every brushing. ${ }^{20}$

According to Komiyama et al. ${ }^{48} \quad 0.12 \%$ chlorhexidine was the most effective disinfectant when toothbrushes contaminated in vitro with standardized suspensions of S. mutans, Streptococcus pyogenes, S. aureus or C. albicans were tested. Nelson-Filho et al. ${ }^{22}$ used $0.12 \%$ chlorhexidine gluconate for disinfection of children's toothbrushes and reported total elimination of $S$. mutans after 20 hours. A study by Nascimento et al. ${ }^{49}$ found $0.12 \%$ chlorhexidine gluconate to be more effective in reducing bacterial colonization on toothbrushes after storage in closed containers. Andolex contains Benzydamine HCL and chlorhexidine gluconate, which kills or inhibits the growth of bacteria, viruses and fungi. ${ }^{50}$

Our study showed Listerine $\AA$ to be as effective as Andolex $(0.12 \%$ chlorhexidine gluconate), but less effective than Brushtox in disinfecting toothbrushes. In contrast, Basman et al. ${ }^{51}$ reported Listerine $\AA$ to be more effective than $0.12 \%$ chlorhexidine gluconate in disinfecting toothbrushes. Konidala et al. ${ }^{52}$ reported $100 \%$ efficacy of Listerine $\AA$ in disinfection of children's toothbrushes. According to Belanger-Giguere et al. $^{54}$ using Listerine $\mathbb{R}$ for 20 minutes to disinfect toothbrushes contaminated in vitro with $\mathrm{S}$. mutans showed lower effectiveness than mouthwashes with chlorhexidine.

Mehta et al. ${ }^{12}$ showed that overnight immersion of toothbrushes in Listerine $\AA$ was less effective than Brushtox in decontaminating toothbrushes. In contrast, Merchan et al. ${ }^{54}$ evaluated the in vitro effectiveness of $0.12 \%$ chlorhexidine gluconate and Listerine $\AA$ in toothbrush disinfection and found Listerine $₫$ less effective than $0.12 \%$ chlorhexidine gluconate. Beneduce et al. ${ }^{55}$ showed that treating toothbrush heads with Listerine ${ }^{\circledR}$ for $7 \mathrm{~min}$ was less effective than hydrogen peroxide in reducing the numbers of both aerobic and anaerobic bacteria.

Essential oil derivates, including Listerine $₫$ are reliable antiseptic and disinfecting agents with no secondary effects on host's oral cavity. ${ }^{6,49}$ Listerine $\AA$ is composed of menthol, ethanol, thymol, eucalyptol (essential oils) and methyl salicylate as active agents. ${ }^{52}$ Listerine ${ }^{\circledR}$ inhibits enzymatic activity of bacteria, disrupts their cell walls, thereby preventing their aggregation and slowing down their multiplication. ${ }^{56}$

Sterile distilled water did not decontaminate any of the toothbrushes and a statistically significant difference was observed when compared with using Brushtox, Listerine ${ }^{\circledR}$ and Andolex $C(p<0.00001)$. This is in agree- 
ment with a study by Nelson Filho et al. ${ }^{46}$ where microbial contamination was detected in $100 \%$ of tooth brushes sprayed with sterile water. Macari et al. ${ }^{45}$ reported that rinsing toothbrushes with sterile water resulted in continued high levels of contamination and biofilm. In another study, uncountable colonies of bacteria were observed after treating toothbrushes with water. ${ }^{57}$ Our study show that the use of water to decontaminate toothbrushes after regular use is ineffective as also reported by other studies. ${ }^{52,53,58}$.

The inability to use molecular methods to detect noncultivable microorganisms remains a limitation of this study. The possibility that the 22 toothbrushes without growth may have been newly purchased by patients or disinfected before they were collected for the study can not be overruled as limitations. It is recommended that sensitive and specific molecular assays be employed to detect, in particular, non-cultivable microorganisms.

\section{CONCLUSION}

Toothbrushes in this study were contaminated with various types of microorganisms, with CoNS predominating. Brushtox was the best disinfectant, reducing the number of contaminating microorganisms on disinfected toothbrushes by $90 \%$ after overnight immersion.

Oral health practitioners should raise awareness to their patients regarding the need to disinfect toothbrushes and that water alone does not disinfect the toothbrushes. The use of Brushtox as a soaking medium should be encouraged for disinfection of toothbrushes between uses.

\section{Acknowledgements}

Wright Millners for providing Oral B toothbrushes which were handed out to patients as a replacement of their used toothbrush. Ivodent for providing Brushtox.

\section{References}

1. Leö H. Oral hygiene in the prevention of caries and periodonta disease. Int Dent J. 2000; 50: 129-39.

2. Choo A, Delac ML, Messer LB. Oral hygiene measures and promotion: Review and considerations. Aust Dent J. 2001; 46: 166-73.

3. Mandel ID: Oral disinfectant agents for controlling plaque and gingivitis. J Clin Periodontol. 1988; 15: 488-98

4. Karibasappa GN, Nagesh L, Sujatha BK. Assessment of microbial contamination of toothbrush head: An in vitro study. Indian J Dent Res. 2011; 22: 2-5.

5. Glass RT, Lare MM. Toothbrush contamination: a potential health risk? Quintessence Int. 1986; 17: 39-42.

6. Taji SS, Rogers AH. The microbial contamination of toothbrushes. A pilot study. Aust Dent J. 1998; 43: 128-30.

7. Fernandez V, Cesar D. Microbiology evaluation of toothbrushes. In Vitro Cell. Dev Biol Anim. 2006; 42: $31 \mathrm{~A}$.

8. Muller HP, Lange DE, Muller RF. Actinobacillus actinomycetemcomitans contamination of toothbrushes from patients harbouring the organism. J Clin Periodontol. 1989; 16: 388-90.

9. Quirynen M, De Soete M, Pauwels M, Gizani S, Van Meerbeek B, van Steenberghe D. Can toothpaste or a toothbrush with antibacterial tufts prevent toothbrush contamination? J Periodontol. 2003; 74: 312-22.
10. Wetzel WE, Schaumburg C, Ansari F, Kroeger T, Sziegoleit A. Microbial contamination of toothbrushes with different principles of filament anchoring. J Am Dent Assoc. 2005; 136: 75864.

11. Mehta A, Sequeira PS, Bhat G. Bacterial contamination and decontamination of toothbrushes after use. N Y State Dent J. 2007; 73: 20-2.

12. ADA. Statement on Toothbrush Care: Cleaning, Storage and Replacement. (Accessed May 2019). Available at: http://www. ada.org/1887.aspx.

13. Goldsmith RN, Shey Z, Houpt MI, Schreiner H, Greenberg B. Toothbrush bristle wear and adherence of Streptococcus mutans. Pediatr Dent. 2007; 29: 243-7.

14. Nascimento C, Scarabel TT, Miani PK, Watanabe E, Pedrazzi V. In vitro evaluation of the microbial contamination on new toothbrushes: a preliminary study. Micro Res Tech. 2012; 75: 42-5.

15. Boylan R, Li Y, Simeonova $L$ et al. Reduction in bacterial contamination of toothbrushes using the Violight ultraviolet light activated toothbrush sanitizer. Am J Dent. 2008; 21: 313-7.

16. Aysegül Ö, Elgin IE, Gulcin A, Nedim S. The efficacy of chlorhexidine spray vs mouthwash in the microbial contamination of child toothbrushes. J Dent Child. 2007; 74: 177-81.

17. Naik R, Ahmed Mujib BR, Telagi N, Anil BS, Spoorthi BR. Contaminated tooth brushes-potential threat to oral and general health. Microsc Res and Techniq. 2015; 75: 42-5.

18. Pesevska S, Ivanovski K, Mindova $S$ et al. Bacterial contamination of the toothbrushes. J Int Dent Med Res. 2016; 9: 6-12.

19. Nascimento AP, Watanabe E, Ito IY. Toothbrush contamination by Candida spp. and efficacy of mouthrinse spray for their disinfection. Mycopathologia. 2010; 169: 133-8.

20. Rodrigues KN, Motter CW, Pegoraro DA, Menoli APV, Menolli RA. Microbiological contamination of toothbrushes and identification of a decontamination protocol using chlorhexidine spray. Rev Odonto Cienc. 2012; 27: 213-7.

21. Susheela P, Radha R. Studies on the micro-biological contamination of toothbrushes and importance of decontamination using disinfectants. WJPMR. 2016; 2: 201-7.

22. Suido H, Offenbacher S, Arnold R. A clinical study of bacterial contamination of chlorhexidine-coated filaments of an Interdental Brush. J Clin Dent. 1998; 9: 105-9.

23. Nelson-Filho P, Macari S, Faria G, Assed S, Ito IY. Microbial contamination of toothbrushes and their decontamination. Pediatr Dent. 2000; 22: 381-4.

24. Vitkov L, Hermann A, Krautgartner WD et al. Chlorhexidine-induced ultrastructural alterations in oral biofilm. Microsc Res Tech. 2005; 68: 85-9.

25. Sammons RL, Kaur D, Neal P. Bacterial survival and biofilm formation on conventional and antibacterial toothbrushes. Biofilms. 2004; 1: 123-30.

26. Frazelle MR, Munro CL. Toothbrush contamination: a review of the literature. Nurs Res Pract. 2012; 420630. doi: 10.1155/2012/420630.

27. Ferreira CA, Savi GD, Panatto AP, Generoso JS, Barichello T. Microbiological evaluation of bristles of frequently used toothbrushes. Dent Press J Orthod. 2012; 17: 72-6.

28. Saini R, Kulkarni V. Toothbrush: A Favorable Media for Bacterial Growth. Int J Experiment Dent Sci. 2013; 2: 27-8.

29. Bannerman TL. Staphylococcus, Micrococcus, and other catalase positive cocci that grow aerobically, In: Murray PR, Baron EJ, Jorgensen JH, Pfaller MA, Yolken RH eds. Manual of Clinical Microbiology, 8th ed., vol. 1. American Society for Microbiology, Washington DC, 2003: 384-404.

30. Otto M. Molecular basis of Staphylococcus epidermidis infections. Semin Immunopathol. 2012; 34: 201-214.

31. Osho A, Thomas BT, Akande YA, Udor RD. Toothbrushes as fomites. J Dent Oral Hyg. 2013; 5: 92-4.

32. Abd-Ulnabi RM. Bacterial contamination of toothbrushes with comparison of healthy and dental patients. Basrah J Sci. 2012; 30: 120-30 
33. Curtis V., Biran A., Deverell K., Hughes C., Bellamy K., Drasar B. Hygiene in the home: relating bugs and behaviour. Social Science \& Medicine. 2003; 57: 657-72.

34. Samuel O, Ifeanyi O. Bacterial contamination of used manual toothbrushes obtained from some students of Nnamdi Azikiwe University Awka, Nigeria. Univ J Micr Res. 2015; 3: 56-9.

35. Osungunna M, Oyajoju O. Used Toothbrushes: Microbial evaluation and antibiotic susceptibility profiles of associated bacteria. Br Microbiol Res. J 2016; 15: 1-9.

36. Costa SF, Miceli MH, Anaissie EJ. Mucosa or skin as source of coagulase-negative Staphylococcal bacteraemia? Lancet Infect Dis. 2004; 4: 278-86.

37. Efstratiou M, Papaioannou W, Nakou M, Ktenas E, Vrotsos IA, Panis V. Contamination of a toothbrush with antibacterial properties by oral microorganisms. J Dent. 2007; 35: 331-7.

38. Lynch JD, Michalek SM, Zhu M, Drake D, Qian F, Banas JA. Cariogenicity of Streptococcus mutans glucan-binding protein deletion mutants. Oral Health Dent Manag. 2013; 12: 191-9.

39. Amarasinghe N, Athavan M, Jayamanne $D$ et al. Bacterial profile and antibiotic susceptibility pattern of adult lower respiratory tract infections in Colombo, Sri Lanka. J Health Soc Sci. 2018; 3: 27-36.

40. World Health Organization. Guidelines for drinking-water quality, fourth edition. Geneva: World Health Organization; 2011. [Online] Available from: http://www.who.int/water_sanitation_ health/publications/2011/dwq_guidelines/en/. Accessed May 2019.

41. Slots J, Jorgensen MG. Effective, safe, practical and affordable periodontal antimicrobial therapy: where are we going, and are we there yet? Periodontol 2000 2002; 28: 298-312.

42. Sukhabogi JR, Chandrashekar BR, Haritha N et al. Qualitative and quantitative assay of microbial contamination of tooth brushes stored in different sanitary settings. Inter J Adv Research 2015; 3: 126-31.

43. Coleman DC, Rinaldi MG, Haynes K et al. Importance of Candida species other than Candida albicans as opportunistic pathogens. Med Mycol. 1998; 36(suppl 1): 156-65

44. Müller HP, Barrieshi-Nusair KM, Könönem E, Yang M. Effect of triclosan copolymer-containing toothpaste on the association between plaque and gingival bleeding: a randomized controlled clinical trial. J Clin Periodontol. 2006 Nov; 33: 811-8.

45. Neal PR, Rippin JW. The efficacy of a toothbrush disinfectant spray - an in vitro study. J Dent. 2003; 31: 153-7.

46. Macari S, Ito IY, Silva RAB, Silva LAB, Paula-Silva FWG. Efficacy of antimicrobial sprays for disinfection of children's toothbrushes - a randomized clinical trial. RPG Rev Pós Grad. 2011; 18: 8-12.

47. Nelson-Filho P, Faria G, Silva RAB, Rossi MA, Ito IY. Evaluation of the contamination and disinfection methods of toothbrushes used by 24-to-48-month-old children. J Dent Child. 2006; 73: 152-8

48. Carter JN, Paterson KMA. Brushtox: Evaluation of disinfectant efficacy [clinical note]. Warwickshire, UK: Research Laboratory; 1998: 6-18.

49. Komiyama EY, Back-Brito GN, Balducci I, Cristiane Yumi Koga-Ito CY. Evaluation of alternative methods for the disinfection of toothbrushes. Braz Oral Res 2010; 24: 28-33.

50. Nascimento C, Sorgini MB, Pita MS et al. Effectiveness of three antimicrobial mouthrinses on the disinfection of toothbrushes stored in closed containers: a randomized clinical investigation by DNA Checkerboard and Culture. Gerodontology. 2014; 31 : 227-36.

51. Van Strydonck DAC, Slot DE, Van der Velden U, Van der Weijden F. Effect of a chlorhexidine mouthrinse on plaque, gingival inflammation and staining in gingivitis patients: a systematic review. J Clin Periodontol. 2012; 39: 1042-55.

52. Basman A, Peker I, Akca G, Alkurt MT, Sarikir C, Celik I . Evaluation of toothbrush disinfection via different methods. Braz Oral Res. 2016; 30: e65.
53. Konidala U, Nuvvula S, Mohapatra A, Nirmala SV. Efficacy of various disinfectants on microbially contaminated toothbrushes due to brushing. Contemp Clin Dent. 2011; 2: 302-7.

54. Belanger-Giguere K, Giguere S, Belanger M. Disinfection of toothbrushes contaminated with Streptococcus mutans. Am J Dent. 2011; 24: 155-8.

55. Merchán I, Merino-Alado RL, Briceño EN, Moronta G, Oviedo M. An in Vitro Effectiveness Evaluation of Chemical Agents for Toothbrushes Disinfection. Pesqui Bras Odontopediatria Clin Integr. 2019; 19: e4296.

56. Beneduce C, Baxter KA, Bowman J, Haines M, Andreana S. Germicidal activity of antimicrobials and VIOlightW Personal Travel Toothbrush Sanitizer: An in vitro study. J Dent 2010; 38: 621- 5 .

57. Nelson Filho P, Pereira MS, De Rossi A et al. Children's toothbrush contamination in day care centers: How to solve this problem? Clin Oral Investig 2014; 18: 1969-74.

58. Jathar P, Panse A, Desai AR. Comparative Evaluation of Various Disinfectant Agents to Disinfect Toothbrush Microbiota. J Pedo Rehabil. 2018; 3: 12-7.

59. Peker I, Akca G, Sarikir C, Toraman Alkurt M, Celik I. Effectiveness of alternative methods for toothbrush disinfection: an in vitro study. Scient World J. 2014; ID726190. doi:10. $1155 / 2014 / 726190$ 